九州大学学術情報リポジトリ

Kyushu University Institutional Repository

\title{
On the statistical decision function I.
}

Mi yasawa, Koichi

Akita University

https://doi.org/10.5109/12947

出版情報 : 統計数理研究. 4 (1/2)，pp. 22-32，1950-12. Research Association of Statistical Sciences

バージョン :

権利関係 : 


\title{
ON THE STATISTICAL DECISION FUNCTION ${ }^{1)}$ I.
}

\author{
Kôichi Miyasawa
}

(Akita Univ.)

\section{Introduction}

Let $X=\left\{X_{i}\right\}(i==1,2,3, \ldots)$ be an infinite sequence of chance variables. Any particular observation $x$ on $X$ is given by a sequence $x=\left\{x_{i}\right\} \quad(i=1,2,3, \ldots)$ of real values, where $x_{i}$ denotes the observed value of $X_{i}$. Let the space of all the sample points $x$ be $M$, the Borel field which contains all the sets $\left\{\boldsymbol{x} ; \boldsymbol{x}_{i}<\boldsymbol{a}_{i}, i=1,2, \ldots\right\}$ be $K$, where $\boldsymbol{a}_{i}$ are real numbers or $+\infty$, and the Lebesgue measure on $K$ be $m$. Suppose that the distribution function $F(x)$ of $X$ is not. known, it is, however, known that $F(x)$ has the probability density function $p(F \mid x)$ and is an element of a given class $Q$ of distribution functions. There is, furthermore, a space $D^{*}$ given whose elements $d$ represent the possible decision that can be made in the problem under consideration. The problem is to construct a function $D=D(x)$ called the statistical decision function, which associates with each sample point $x$ an element $d$ of $D^{*}$ so that the decision $d=D(x)$ is made when $x$ is observed. Let $W(F, d)$ be the loss suffered by the statistician when $F$ is the true distribution of $X$ and the aecision $d$ is made. We assume that $W(F, d)$ is a non-negative bounded measurable function of $F$ and $d$. Let $c(n)$ be the cost of making $n$ observations, i. e., $c(n)$ is the cost of observing the values of $x_{1}, \ldots, x_{i v}$. Thus, when the true distribution function of $X$ is $F$, and if we decide the element of $D^{*}$ by the decision function $d_{n}(x)$ which depends only on the first $n$ coordinates $x_{1}, x_{2}, \ldots, x_{n}$ of the sample $x$, the loss is given by the following sum

$$
r\left(F, d_{n}(x)\right)=W\left(F, d_{n}(x)\right)+c(n) .
$$

$A$ sequential statistical decision function $D$ is composed of the following two sequences $B=\left\{B_{j}\right\}$ and $D=\left\{d_{j}\right\}$.

(i) $\left\{B_{j}\right\}$ is the sequence of $B_{0}$ and disjoint subsets $B_{1}, B_{2}, \ldots B_{j}, \ldots$ of $M$, where $B_{j}$ depends on the first $j$ coordinates $x_{1}, \ldots, x_{j}$ of a sample $x$ and $B_{j}$ indicates that the sampling should stop at the $j$-th observation when $x \in B_{j}$ $(j=1,2, \ldots), B_{0}$ is the event that we do not sample at all, but take some decision immediately and it will have probability either 0 or 1 . It should be

$$
\sum_{j=0}^{\infty} P_{r}\left(B_{j} \mid F\right)=1 \text { for all } F \in Q .
$$

This sequence $B=\left\{B_{j}\right\} \quad(j=0,1,2, \ldots)$ is called a sequential procedure.

(ii) $\left\{d_{j}\right\}$ is the sequence of $d_{0}$ and decision functions $d_{1}(x), d_{2}(x), \ldots$,

1) Communicated at the Autumn-Meeting of Japanese Math. Soc., at Kôbe Univ., October $20,1950$. 
$d_{j}(x), \ldots$, where $d_{0}$ is an element of $D^{*}$ and $d_{j}(x)$ is the function of the first $j$ coordinates $x_{1}, \ldots, x_{j}$ of $x \in B_{j}$ and its value is some decision, i.e., some element of $D^{*}$. This sequence $D=\left\{d_{j}\right\}$ is also called a decision function.

The sequential decision function $D$ which is determined by two sequence $B$ and $D$ will be denoted by $D=(B, D)$. Then the average loss caused by the sequential decision function $\underline{D}$ when $F$ is the true distribution function of $X$ is given by

$$
\boldsymbol{r}(F, \underline{D})=\sum_{j=0}^{\infty} \int_{B j} \boldsymbol{r}\left(F, d_{j}(x)\right) p(F \mid x) d x .
$$

Here, we assume that the series on the right hand member of (1) is always convergent in our problem. Let $\xi$ be an a priori distribution on $Q$, i. e., $\xi$ is a probability measure defined over a suitably chosen Borel field of subsets of $Q$.

Then the expected value of $r(F, D)$ is given by

$$
r(\xi, \underline{D})=\int_{\Omega} r(F, D) d \xi
$$

$r(\xi, D)$ is called the risk when $\xi$ is the a priori distribution on $\Omega$ and $D$ is the decision function adopted. The sequential decision function $D^{*}$ is called a Bayes solution relative to the a priori distribution $\xi$, if

$$
r\left(\xi, D^{*}\right)=\inf _{D} r(\xi, \underline{D}) \text {. }
$$

Our object is to determine the necessary and sufficient condition so that a sequential decision function will be a Bayes solution relative to the a priori distribution $\xi$.

\section{Theorem and its Proof}

Let $D_{j}^{*}$ be the set of all decision functions $d_{j}(x)$ which depends only on the first $j$ coordinates $x_{1}, \ldots, x_{j}$ of $x(j=1,2, \ldots)$. For $j=0, d_{0}$ is some element of $D^{*}$, i. e., $D_{0}^{*}=D^{*}$. Let $\left\{d_{j_{n}}\right\}$ and $d_{j}^{*}$ be a sequence of elements of $D_{j}{ }^{*}$ and an element of $D_{j}^{*}$, respectively. If it is valid that

$$
W\left(F, d_{j_{3}}(x)\right) \rightarrow W\left(F, d_{j}^{*}(x)\right),
$$

as $n \rightarrow \infty$, for all $F \in \Omega$ all $x \in M$, then we say that $\left\{d_{j_{n}}\right\}$ converges to $d_{j}^{*}$. We assume that $D_{j}^{*}$ is compact in the sense of the convergence of the above definition.

LEMMA.

For any a priori distribution $\xi$ of $F$ and for any $j$, there exists a decision function $d_{j}^{\circ}(x)$ such that

$$
r\left(\xi, d_{j}{ }^{\circ}(x)\right)=\inf _{a j \in D j *} r\left(\xi, d_{j}(x)\right) \quad \text { for all } x .
$$

where $d_{j}(x)$ is an element of $D_{j}$, and 


$$
r\left(. \xi, d_{j}(x)\right)=\int_{\Omega} r\left(F, d_{j}(x)\right) p(F \mid x) d \xi .
$$

As this lemma will be proved in the same way as done in GIRSHICK's'), we do not refer to it here.

We write

$$
r_{j}(\xi, x)=r_{i}\left(\xi, d_{j}^{\circ}(x)\right)=\inf _{a j} r\left(\xi, d_{j}(x)\right)
$$

Let $N$ be any fixed integer, and by the induction backwards we define functions $\alpha_{j N}(\xi, x)(j=0,1, \ldots, N)$ which depends only on the first $j$ coordinates of $x$.

That is

$$
\alpha_{N N}(\xi, x)=r_{N}(\xi, x)
$$

and, for $j<N$

$$
\alpha_{j N}(\xi, x)=\min \left(r_{j}(\xi, x), E_{j}\left\{\alpha_{j+1, N}(\xi, x)\right\}\right)
$$

where $E_{j}$ is the conditional expectation given $x_{1}, \ldots, x_{j}$, i. e.,

$$
E_{j}\left\{\alpha_{j+1, N}(\xi, x)\right\}=\int_{-\infty}^{\infty} \alpha_{j+1, N}(\xi, x) d x_{j+1} .
$$

It can be seen easily that when $j$ is fixed, $\alpha_{j N}(\xi, x)$ is non-negative and non-increasing as $N$ increases. Therefore there exists $\lim _{N \rightarrow \infty} \alpha_{j N}(\xi, x)$, and we represent this limit as $\alpha_{j}(\xi, x)$, i. e.,

$$
\alpha_{j}(\xi, x)=\lim _{N \rightarrow \infty} \alpha_{j N}(\xi, x) .
$$

Then the following relation will hold

$$
\alpha_{j}(\xi, x)=\min \left\{r_{j}(\xi, x), E_{j}\left\{\alpha_{j+1}(\xi, x)\right\}\right\},
$$

and consequently

$$
\alpha_{j}(\xi, x) \leqq r_{j}(\xi, x) \quad(j=0,1,2, \ldots) .
$$

By means of these functions $\alpha_{j}(\xi, x)$ we define subsets $S_{j}$ of $M$ as follows:

$$
S_{j}=\left\{x ; r_{i}(\xi, x)>\alpha_{i}(\xi, x) \text { for } i<j \text {, and } r_{j}(\xi, x)=\alpha_{j}(\xi, x)\right\} \text {. }
$$

It is clear that thus defined sequence of subsets $\left\{S_{j}\right\} \quad(j=0,1,2, \ldots)$ forms a sequential procedure.

Let us denote the sequential procedure which consists of the sequence $\left\{S_{j}\right\}$ by $S_{\xi}$ and the decision function which consists of the sequence $\left\{d_{j}^{\circ}\right\}$ by $D^{\prime}$. Let $D_{\xi}$ be the sequential decision function which is determined by $S_{\xi}$ and $D^{\circ}$, that is,

$$
D_{\xi}=\left(S_{\xi}, D^{\circ}\right)
$$


We assume that if for any fixed element $x$ of some subset $A \subset M$,

$$
r\left(\xi, d_{j}(x)\right)=\int_{\Omega 2} r\left(F, d_{j}(x)\right) p(F \mid x) d \xi
$$

has the minimum value with respect to $d_{j}$, for $d_{j}=d_{j}{ }^{\circ}$ and $d_{j}=d_{j}{ }^{*}$, then it holds $d_{j}^{\circ}(x)=d_{j}^{*}(x)$ almost everywhere on $A$.

Then the following theorem holds.

THEOREM .

There exists a Bayes solution relative to any a priori distribution $\xi$. The necessary and sufficient condition for that the sequential decision function $D=(T, D)$, where $T=\left\{B_{j}\right\}, D=\left\{d_{j}\right\}$, will be a Bayes solution relative to the a priori distribution $\xi$ is that

(i) $d_{j}(x)=d_{j}^{\circ}(x)$, almost everywhere on $B_{j},(j=0,1,2, \ldots)$

(ii) the following relation holds except for a set of measure 0

$$
B_{0}=S_{0}, B_{1} \subset S_{1}, B_{2} \subset S_{1}+S_{2}, \ldots, B_{j} \subset S_{1}+S_{2}+\ldots+S_{j}, \ldots
$$

Consequently, if we write

$$
B_{i} \cap S_{i}=D_{i}, S_{i}-D_{i}=S_{i}{ }^{\prime}, B_{l} \cap S_{k}{ }^{\prime}=B_{l}{ }^{k}(l=2,3, \ldots ; k=1,2, \ldots l-1)
$$

then it holds

$$
B_{1}=D_{1}, B_{2}=D_{2}+B_{2}{ }^{1}, B_{3}=D_{3}+B_{3}{ }^{1}+B_{3}{ }^{2}, \ldots, B_{j}=D_{j}+B_{j}{ }^{1}+B_{j}{ }^{2}+\ldots+B_{j}{ }^{j-1}, \ldots
$$

and

$$
S_{i}{ }^{\prime}=B_{i+1}^{i}+B_{i+2}^{i}+B_{i+3}^{i}+\ldots,(i=1,2,3, \ldots) .
$$

(iii) if there exists some $k$ such that $m\left(S_{k}{ }^{\prime}\right)>0$, then except for the set of measure 0 it holds

$$
\boldsymbol{r}_{k+1}(\xi, x)=E_{k+1}\left\{\boldsymbol{r}_{k+2}(\xi, x)\right\},
$$

on the complement $C B_{k+1}^{k}$ of $B_{k+1}^{k}$ with respect to $S_{k}{ }^{\prime}$

$$
\boldsymbol{r}_{k+2}(\xi, x)=E_{k+2}\left\{\boldsymbol{r}_{k+3}(\xi, x)\right\},
$$

on the complement $C\left[B_{k+1}^{k}+B_{k+2}^{k}\right]$ of $\left[B_{k+1}^{k}+B_{k+2}^{k}\right]$ with respect to $S_{k}^{\prime}$, and so on.

PROOF: It can be shown that the above defined sequential decision function $D_{\xi}=\left(S_{\xi}, D^{\circ}\right)$ is a Bayes solution relative to the a priori distribution $\xi$ in a analogous way as done in Girshick's $\left.{ }^{1}\right)$, so we will omit it here. Let $\underline{D}=(T$, $D), T=\left\{B_{j}\right\}, D=\left\{d_{j}\right\}$ be a Bayes solution relative to the a priori distribution $\xi$.

At first we will prove that the condition (i) is necessary. It follows from the lemma that

$$
r\left(\xi, d_{j}(x)\right) \geq r\left(\xi, d_{j}^{\circ}(x)\right)
$$


Now let us put

$$
R_{j}=\left\{x ; x \in B_{j} \text { and } r\left(\xi, d_{j}(x)\right)>r\left(\xi, d_{j}{ }^{\circ}(x)\right)\right\},
$$

and suppose that $m\left(R_{j}\right)>0$.

Then, we can choose $\delta>0$ and $R_{j}{ }^{\prime} \subset R_{j}$ such that $m\left(R_{j}{ }^{\prime}\right)>0$, and the following relation holds for $x \in R_{j}^{\prime}$

$$
r\left(\xi, d_{j}(x)\right)>r\left(\xi, d_{j}^{\circ}(x)\right)+o .
$$

Consequently it follows

$$
\begin{aligned}
r\left(\xi ; B_{j}, d_{j}\right) & =\int_{B j} \int_{\Omega} r\left(F, d_{j}(x)\right) p(F \mid x) d \xi d x=\int_{B j} r\left(\xi, d_{j}(x)\right) d x \\
& >\int_{R^{\prime} j} r\left(\xi, d_{j}^{\circ}(x)\right) d x+o \int_{R^{\prime} j} d x+\int_{C K^{\prime} j} r\left(\xi, d_{j}{ }^{\circ}(x)\right) d x \\
& =\int_{B j} r\left(\xi, d_{j}^{\circ}(x)\right) p x+\delta m\left(R_{j}^{\prime}\right) \\
& >\int_{B j} r\left(\xi, d_{j}{ }^{\circ}(x)\right) d x=r\left(\xi ; B_{j}, d_{j}^{\circ}\right) .
\end{aligned}
$$

(where $C R_{j}{ }^{\prime}$ denotes the complement of $R_{j}{ }^{\prime}$ with respect to $B_{j}$ ) And generally it holds

$$
r\left(\xi ; B_{i}, d_{i}\right) \geqq r\left(\xi ; B_{i}, d_{i}^{\circ}\right) .
$$

Consequently, it folds

$$
r(\xi, \underline{D})=\sum_{j=0}^{\infty} r\left(\xi, B_{j}, d_{j}\right)>\sum_{j=0}^{\infty} r\left(\xi ; B_{j}, d_{j}{ }^{\circ}\right)=r\left(\xi ; T, D^{\circ}\right)
$$

Thus we have a sequential decision function $\underline{D}^{\prime}=\left(T, D^{\circ}\right)$ such that $r(\xi, \underline{\mathrm{D}})$ $>r\left(\xi, \underline{D}^{\prime}\right)$. This contradicts with the assumption that the sequential decision function $\underline{D}=(T, D)$ is a Bayes solution relative to $\xi$.

Therefore, it holds on $B_{j}$

$$
r\left(\xi, d_{j}(x)\right)=r\left(\xi, d_{j}{ }^{\circ}(x)\right)(j=0,1,2, \ldots)
$$

except for a set of measure 0 . Consequently it follows from our assumption that $d_{j}(x)=d_{j}{ }^{\circ}(x)$ almost everywhere on $B_{j}(j=0,1,2, \ldots)$.

Next we will prove that the condition (ii) is necessary.

We put

$$
B_{i}^{\prime}=\left(B_{i}-D_{i}\right) \cap C\left[S_{1}+S_{2}+\ldots+S_{i}\right],
$$

where $C\left[S_{1}+\ldots+S_{i}\right]$ is the complement of $\left[S_{1}+\ldots+S_{i}\right]$ with respect to $M$, 


$$
\begin{gathered}
B_{i}{ }^{\prime \prime}=\left(B_{i}-D_{i}\right) \cap\left[S_{1}+S_{2}+\ldots+S_{i}\right] \\
S_{i}{ }^{l}=B_{l}{ }^{\prime} \cap S_{i}(i=l+1, l+2, \ldots ; l=1,2, \ldots)
\end{gathered}
$$

Let $S_{i}{ }^{l}(1,2, \ldots, l)$ be the intersection of $S_{i}{ }^{l}$ and the subset $C\left(x_{1}, \ldots, x_{l}\right)$ of $M$ defined by $x_{1}=$ const.,..., $x_{l}=$ const. and $C S_{i}{ }^{l}(1,2, \ldots, l)$ be the complement of $S_{l}(1,2, \ldots, l)$ with respect to $C\left(x_{1}, \ldots, x_{l}\right)$.

To prove that $m\left(B_{i}^{\prime}\right)=0 \quad(i=1,2, \ldots)$, we assume that $m\left(B_{l}^{\prime}\right)>0$ for some $l$.

Now, by the definition of $S_{j}$, it follows that if $x \in B_{l}^{\prime}$

$$
\begin{aligned}
r_{l}(\xi, x)> & \alpha_{l}(\xi, x)=E_{l}\left\{\alpha_{l+1}(\xi, x)\right\}=\int_{-\infty}^{\infty} \alpha_{l+1}(\xi, x) d x_{l+1} \\
& =\int_{S_{l+1}^{l}(1,2, \ldots, l)} \alpha_{l+1}(\xi, x) d x_{l+1}+\int_{c_{s_{l+1}^{l}(1,2 \ldots, l)}} \alpha_{l+1}(\xi, x) d x_{l+1} .
\end{aligned}
$$

And if $x \in S_{l+1}^{\prime}(1,2, \ldots, l)$, then we have

$$
\alpha_{l+1}(\xi, x)=r_{l+1}(\xi, x) .
$$

Therefore it follows

$$
r_{l}(\xi, x)>\int_{S_{l+1}^{l}(1,2, \ldots, l)} r_{l+1}(\xi, x) d x_{l+1}+\int_{C s_{l+1}^{l}(1,2, \ldots, l)} \alpha_{l+1}(\xi, x) d x_{l+1} .
$$

If $x \in C S_{l+1}^{l}(1,2, \ldots, l)$, it holds by the definition of $S_{j}$

$$
r_{l+1}(\xi, x)>\alpha_{l+1}(\xi, x)
$$

hence

$$
\alpha_{l+1}(\xi, x)=E_{l+1}\left\{\alpha_{l_{+}}(\xi, x)\right\} \text {. }
$$

Consequently it follows

$$
\begin{aligned}
& \text { (2.33) } \int_{C S_{l+1}^{l}(1,2, \ldots, l)} \alpha_{l+1}(\xi, x) d x_{l+1}=\int_{C S_{l+1}^{l}(1,2, \ldots, l)} \int_{-\infty}^{\infty} \alpha_{l+2}(\xi, x) d x_{l+2} d x_{l+1} \\
& =\int_{s_{l+2}^{l}(1,2, \ldots, l)} \int_{-\infty}^{\infty} \alpha_{l+2}(\xi, x) d x_{l+2} d x_{l+1}+\int_{\left.C s_{l+1}^{l}(1,2, \ldots, l)+s_{l+2}^{l}(1,2, \ldots, l)\right]} \int_{-\infty}^{\infty} \alpha_{l+2}(\xi, x) d x_{l+2} d x_{l+1}
\end{aligned}
$$$$
=\int_{\left.s_{l+2}^{l} 1,2, \ldots, l\right)} \int_{s_{l+2}^{l}(1,2, \ldots, l, l+1)} \alpha_{l+2}(\xi, x) d x_{l+2} d x_{l+1}
$$$$
+\int_{\left.s_{l+2^{2}}^{l}, \ldots l\right)} \int_{C s_{l+2}^{l}(1,2, \ldots l, l+1)} \alpha_{l+2}(\xi, x) d x_{l+2} d x_{l+1}
$$$$
+\int_{\left(t-s_{l+1}^{l}(1, \ldots l)+s_{l+2}^{l}(, \ldots,)\right]} \int_{-\infty}^{\infty} \alpha_{l+2}(\xi, x) d x_{l+2} d x_{l+1}
$$

$$
=\int_{s_{l+2^{l}}^{l}(1, \ldots, l)} r_{l+2}(\xi, x) d x_{l+2} d x_{l+1}+\int_{C_{i s}^{\prime} s_{l+1}^{l}\left(1, \ldots, l,+s_{l+2^{2}}^{l}, \ldots, l_{l+2}\right.} \alpha_{l+2}(\xi, x) d x_{l+2} d x_{l+1} \text {. }
$$


Since we have

$$
\alpha_{l+2}(\xi, x)=r_{l+2}(\xi, x) \text { on } S_{l+2}^{l}(1, \ldots, l, l+1) .
$$

From (2.31) and (2.33) it follows

$$
\begin{aligned}
r_{l}(\xi, x)> & \int_{s_{l+1}^{l}(1, \ldots, l)} r_{l+1}(\xi, x) d x_{l+1}+\int_{s_{l+2}^{l}(1, \ldots, l)} r_{l+2}(\xi, x) d x_{l+2} d x_{l+1} \\
& +\int_{c\left[s_{l+1}^{l}(1, \ldots, l)+s_{l+2}^{l}(1, \ldots, l)\right]} \alpha_{l+2}(\xi, x) d x_{l+2} d x_{l+1} \cdot
\end{aligned}
$$

If $x \in B_{l}{ }^{\prime}$, using the analogous method as above, we can conclude the following relation,

$$
\begin{gathered}
r_{l}(\xi, x)>\int_{s_{l+1}^{l}(1, \ldots, l)} r_{l+1}(\xi, x) d x_{l+1}+\int_{s_{l+2}^{l}(1, \ldots, l)} r_{l+2}(\xi, x) d x_{l+2} d x_{l+1} \\
+\int_{s_{l+3}^{l}(1, \ldots, l)} r_{l+3}(\xi, x) d x_{l+3} d x_{l+2} d x_{l+1}+\ldots .
\end{gathered}
$$

Therefore, if $\boldsymbol{m}\left(B_{l}{ }^{\prime}\right)>0$, we have

$$
\int_{B_{l}^{\prime}} \boldsymbol{r}_{l}(\xi, x) d x>\int_{s_{l+1}^{l}} r_{l+1}(\xi, x) d x+\int_{s_{l+2}^{l}} r_{l+2}(\xi, x) d x+\int_{S_{l+3}^{l}} r_{l+3}(\xi, x) d x+\ldots
$$

Now, we construct the sequential procedure $T^{*}=\left\{C_{j}\right\}$ as follows:

$C_{0}=B_{0}, C_{1}=B_{1}, \ldots, C_{l-1}=B_{l-1}, C_{l}=D_{l}+B_{l}^{\prime \prime} C_{l+1}=B_{l+1}+S_{l+1}^{l}, C_{l+2}=B_{l+2} S_{l+2}^{l}, \ldots$.

Then, it is clear that any two sets $C_{i}$ and $C_{j}(i \neq j)$ are disjoint, and

$$
\begin{aligned}
C_{1}+ & C_{2}+C_{3}+\ldots \\
& =B_{1}+\ldots+B_{l-1}+\left(D_{l}+B_{l}^{\prime \prime}+S_{l+1}^{l}+S_{l+2}^{l}+\ldots\right)+B_{l+1}+B_{l+2}+\ldots \\
& =M .
\end{aligned}
$$

Therefore $T^{*}=\left\{C_{j}\right\}$ is certainly a sequential procedure. Then, for the sequential decision function $D^{*}$, which is determined by $T^{*}$ and $D^{r}$, we have

$$
\begin{aligned}
r\left(\xi, D^{*}\right)=r_{0} P_{r}\left(C_{0}\right)+\int_{C_{1}} r_{1}(\xi, x) d x+\ldots+\int_{C_{l+1}} r_{l-1}(\xi, x) d x \\
\quad+\int_{C_{l}} r_{l}(\xi, x) d x+\int_{C_{l+1}} r_{l+1}(\xi, x) d x+\ldots \\
=r_{0} P_{r}\left(B_{0}\right)+\int_{B_{1}} r_{l}(\xi, x) d x+\ldots+\int_{B_{l-1}} r_{l-1}(\xi, x) d x+\int_{l_{l}+B_{l}^{\prime \prime}} r_{l}(\xi, x) d x \\
+\int_{B_{l+1}+s_{l+1}^{l}} r_{l+1}(\xi, x) d x+\int_{B_{l+2}+s_{l+2}^{l}} r_{l+2}^{l}(\xi, x) d x+\ldots
\end{aligned}
$$




$$
\begin{aligned}
=\boldsymbol{r}_{0} \boldsymbol{P}_{r}\left(B_{0}\right) & +\int_{B_{1}} \boldsymbol{r}_{1}(\xi, x) d x+\ldots+\int_{B_{l-1}} \boldsymbol{r}_{l-1}(\xi, x) d x \\
& +\left\{\int_{B_{l+B_{l^{\prime}}}} \boldsymbol{r}_{l}(\xi, x) d x+\int_{S_{l+1}^{l}} \boldsymbol{r}_{l+1}(\xi, x) d x+\int_{S_{l+2}^{l}} \boldsymbol{r}_{l+2}(\xi, x) d x+\ldots\right\} \\
& +\int_{B_{l+1}} \boldsymbol{r}_{l+1}(\xi, x) d x+\int_{B_{l+2}} \boldsymbol{r}_{l+2}(\xi, x) d x+\ldots
\end{aligned}
$$

So that, from (2.35) and (2.38), it follows

$$
\begin{aligned}
r\left(\xi, D^{*}\right)<r_{0} & P_{r}\left(B_{0}\right)+\int_{B_{1}} r_{1}(\xi, x) d x+\ldots+\int_{B_{l-1}} r_{l-1}(\xi, x) d x \\
& +\left\{\int_{D_{l}+B_{l^{\prime}}} r_{l}(\xi, x) d x+\int_{B_{l^{\prime}}} \boldsymbol{r}_{l}(\xi, x) d x\right\} \\
& +\int_{B_{l+1}} r_{l+1}(\xi, x) d x+\int_{B_{l+2}} r_{l+2}(\xi, x) d x+\ldots \\
= & r\left(\xi ; T, D^{\circ}\right) .
\end{aligned}
$$

That is $r\left(\xi, \underline{D}^{*}\right)<\boldsymbol{r}\left(\xi ; T, D^{\circ}\right)$. This contradicts with the assumption that the sequential decision function $\left(T, D^{\circ}\right)$ is a Bayes solution relative to the a priori distribution $\xi$. Consequently we may conclude $\boldsymbol{m}\left(\boldsymbol{B}_{l}{ }^{\prime}\right)=0 \quad(l=1$, $2, \ldots)$. This shows that the condition (ii) is necessary.

Lastly we will prove that the condition (iii) is necessary.

Let us assume that for some $k$, say $k=1, m\left(S_{1}^{\prime}\right)>0$. If $n \geqq N$, it can be shown easily that $r_{N}(\xi, x) \geqq E_{N}\left\{r_{n}(\xi, x)\right\}$, so that

$$
r_{1}(\xi, x) \geqq E_{1}\left\{r_{2}(\xi, x)\right\} \text {. }
$$

On the other hand, if $x \in S_{1}^{\prime}$, we have $r_{1}(\xi, x)=\alpha_{1}(\xi, x)$, so that

$$
r_{1}(\xi, x) \leqq E_{1}\left\{\alpha_{2}(\xi, x)\right\} \leqq E_{1}\left\{r_{2}(\xi, x)\right\} \text {. }
$$

From (2.40) and (2.41), it follows

$$
r_{1}(\xi, x)=E_{1}\left\{r_{2}(\xi, x)\right\}, \text { if } x \in S_{1}^{\prime} .
$$

Accordingly we have

$$
\int_{S_{1^{\prime}}} r_{1}(\xi, x) d x_{1}=\int_{s_{1}^{\prime}} \int_{-\infty}^{\infty} r_{2}(\xi, x) d x_{2} d x_{1} .
$$

Let $B_{l}{ }^{\prime}(1, \ldots, k)$ be the intersection of $B_{l}{ }^{\prime}$ and $C\left(x_{1}, \ldots, x_{k}\right)$, and let $C B_{l}{ }^{\prime}$ $(1,2, \ldots, k)$ be the complement of $B_{l}{ }^{\prime}(1, \ldots, k)$ with respect to $C\left(x_{1}, \ldots, x_{k}\right)$, where $C\left(x_{1}, \ldots, x_{k}\right)$ is the subset of $M$ defined by $x_{1}=$ const.,., $x_{k}=$ const: Then 


$$
\int_{-\infty}^{\infty} r_{2}(\xi, x) d x_{2}=\int_{B_{2}^{\prime}(\mathrm{I})} r_{2}(\xi, x) d x_{2}+\int_{r_{2}^{\prime}(1)} r_{2}(\xi, x) d x_{2}
$$

So that (2.43) can be written as follows,

$$
\int_{S_{1}^{\prime}} r_{1}(\xi, x) d x_{1}=\int_{B_{2}^{\prime}} r_{2}(\xi, x) d x_{2} d x_{1}+\int_{C B_{2}^{\prime}} r_{2}(\xi, x) d x_{2} d x_{1},
$$

where $C B_{2}{ }^{\prime}$ is the complement of $B_{2}{ }^{\prime}$ with respect to $S_{1}{ }^{\prime}$. Generally it holds that

$$
\left.\left.r_{2}(\xi, x) \geqq E_{2}\right\} r_{3}(\xi, x)\right\},
$$

but now we assume that the condition (iii) does not hold and on some subset of positive measure of $C B_{2}^{\prime}$ it holds

$$
r_{2}(\xi, x)>E_{2}\left\{r_{3}(\xi, x)\right\} \text {. }
$$

Then, it follows

$$
\int_{C B_{2}^{\prime}} r_{2}(\xi, x) d x_{2} d x_{1}>\int_{C B_{2}^{\prime}} \int_{-\infty}^{\infty} r_{3}(\xi, x) d x_{3} d x_{2} d x_{1}
$$

Now

$$
\begin{aligned}
& \int_{C B_{2}^{\prime}(1)} \int_{-\infty}^{\infty} r_{3}(\xi, x) d x_{3} d x_{2}= \int_{B_{3}^{\prime}(1)} \int_{-\infty}^{\infty} r_{3}(\xi, x) d x_{3} d x_{2} \\
&+\int_{C\left[B_{2}^{\prime}(1)+B_{3}^{\prime}(1)\right]} \int_{-\infty}^{\infty} r_{3}(\xi, x) d x_{3} d x_{2} \\
&=\int_{B_{3}^{\prime}(1)} \int_{B_{3}^{\prime}(1,2)} r_{3}(\xi, x) d x_{3} d x_{2}+\int_{B_{3}^{\prime}(1)} \int_{C B_{3}^{\prime}(1,2)} r_{3}(\xi, x) d x_{3}^{\circ} d x_{2} \\
&+\int_{C\left[B_{2}^{\prime}(1)+B_{3}^{\prime}(1)\right]} \int_{-\infty}^{\infty} r_{3}(\xi, x) d x_{3} d x_{2} \\
&=\int_{B_{3}^{\prime}(1)} r_{3}(\xi, x) d x_{3} d x_{2}+\int_{C\left[B_{2}^{\prime}(1)+B_{3}^{\prime}(1)\right]} r_{3}(\xi, x) d x_{3} d x_{2} .
\end{aligned}
$$

Therefore it follows from (2.44), (2.47) and (2.48)

$$
\int_{s_{1}^{\prime}} r_{1}(\xi, x) d x_{1}>\left.\right|_{B_{2}^{\prime}} r_{2}(\xi, x) d x+\int_{B_{3}^{\prime}} r_{3}(\xi, x) d x+\int_{C\left[B_{2}^{\prime}+B_{3}^{\prime} 3\right.} r_{3}(\xi, x) d x,
$$

where $C\left[B_{2}{ }^{\prime}+B_{3}{ }^{\prime}\right]$ is the complement of $B_{2}{ }^{\prime}+B_{3}{ }^{\prime}$ with respect to $S_{1}{ }^{\prime}$. Generally it holds that

$$
r_{3}(\xi, x) \geqq E_{3}\left\{r_{4}(\xi, x)\right\},
$$


but now we assume that on some subset of positive measure of $C\left[B_{2}{ }^{\prime}+B_{3}{ }^{\prime}\right]$ it holds

$$
r_{3}(\xi, x)>E_{3}\left\{r_{4}(\xi, x)\right\}
$$

Then it follows

$$
\int_{C\left[B_{2}^{\prime}+B_{3}^{\prime}\right]} r_{3}(\xi, x) d x>\int_{C\left[B_{2}^{\prime}+B_{3}^{\prime}\right]} \int_{-\infty}^{\infty} r_{4}(\xi, x) d x_{4} d x_{3} d x_{2} d x_{1} .
$$

Let $C\left[B_{2}{ }^{\prime}+B_{3}{ }^{\prime}\right](1,2)$ be the intersection of $C\left[B_{2}{ }^{\prime}+B_{3}{ }^{\prime}\right]$ and $C\left(x_{1}, x_{2}\right)$, etc. Then we have

$$
\begin{aligned}
& \int_{C\left[B_{2}^{\prime}+B_{3}^{\prime}(1,2)\right.} \int_{-\infty}^{\infty} r_{4}(\xi, x) d x_{4} d x_{3} \\
& =\int_{B_{4}^{\prime}(1,2)} \int_{-\infty}^{\infty} r_{4}(\xi, x) d x_{4} d x_{3}+\int_{C\left[B_{2}^{\prime}+B_{3}^{\prime}+B_{4}^{\prime}(1,2)\right.} \int_{-\infty}^{\infty} r_{4}(\xi, x) d x_{4} d x_{3} \\
& =\int_{B_{4}^{\prime}(1,2)} \int_{B_{4}^{\prime}(1,2,3)} r_{4}(\xi, x) d x_{4} d x_{3}+\int_{B_{4}^{\prime}(1,2)} \int_{C B_{4}^{\prime}(1,2,3)} r_{4}(\xi, x) d x_{4} d x_{3} \\
& \quad+\int_{C\left[B_{2}^{\prime}+B_{3}^{\prime}+B_{4}^{\prime}\right](1,2)} \int_{-\infty}^{\infty} r_{4}(\xi, x) d x_{4} d x_{3}
\end{aligned}
$$

Consequently

$$
\int_{C\left[R_{2}^{\prime}+B_{3}^{\prime}\right]} \int_{-\infty}^{\infty} r_{4}(\xi, x) d x_{4} d x_{3} d x_{2} d x_{1}=\int_{B_{4}^{\prime}} r_{4}(\xi, x) d x+\int_{C\left[B_{2}^{\prime}+B_{3}^{\prime}+B_{4}^{4}\right]} r_{4}(\xi, x) d x .
$$

From (2.49), (2.52) and (2.54), it follows

$$
\begin{aligned}
\int_{S_{1}^{\prime}} r_{1}(\xi, x) d x>\int_{B_{2}^{2}} r_{2}(\xi, x) d x+\int_{B_{3}^{\prime}} r_{3}(\xi, x) d x & +\int_{R_{4}^{4}} r_{4}(\xi, x) d x \\
& +\int_{C \bar{B}_{2}^{\prime}+B_{3}^{\prime}+B_{4}^{\prime}} r_{4}(\xi, x) d x .
\end{aligned}
$$

Thus proceeding as above, if the condition (iii) is not satisfied for $k=1$, then we have

$$
\int_{S_{1}^{\prime}} r_{1}(\xi, x) d x>\int_{B_{2}^{\prime}} r_{2}(\xi, x) d x+\int_{B_{3}^{\prime}} r_{3}(\xi, x) d x+\ldots+\int_{B_{l}^{\prime}} r_{l}(\xi, x) d x+\ldots
$$

If the condition (iii) is satisfied here, the left and the right hand members of (2.56) are equal.

As when $k=1$, we have generally 


$$
\int_{S_{k}^{\prime}} \boldsymbol{r}_{k}(\xi, x) d x \geqq \int_{B_{k+1}^{k}} \boldsymbol{r}_{k+1}(\xi, x) d x+\int_{B_{k+2}^{k}} \boldsymbol{r}_{k+2}(\xi, x) d x+\ldots
$$

On the otherhand, we have

$$
\begin{gathered}
D_{i}+S_{i}{ }^{\prime}=S_{i} \quad(i=1,2, \ldots) \\
D_{1}=B_{1}, D_{i}+B_{i}{ }^{1}+B_{i}{ }^{2}+\ldots+B_{i}^{i-1}=B_{i}(i=2,3, \ldots) .
\end{gathered}
$$

Therefore if the condition (iii) is not satisfied for some $k$, say $k=1$, it follows from (2.56), (2.57) and (2.58)

$$
\begin{aligned}
& r\left(\xi ; S_{\xi}, D^{\circ}\right)=r_{0} P_{r}\left(S_{0}\right)+\left[\int_{D_{1}} r_{1}(\xi, x) d x+\int_{S_{1}^{\prime}} r_{1}(\xi, x) d x\right] \\
& +\left[\int_{D_{2}} r_{2}(\xi, x) d x+\int_{S_{2}^{\prime}} r_{2}(\xi, x) d x\right]+\ldots \\
& >r_{0} P_{r}\left(S_{0}\right)+\int_{D_{1}} r_{1}(\xi, x)+\left[\int_{D_{2}} r_{2}(\xi, x) d x+\int_{B_{2}^{2}} r_{2}(\xi, x) d x\right] \\
& +\left[\iint_{D_{3}} r_{3}(\xi, x) d x+\int_{B_{3}^{1}} r_{3}(\xi, x) d x+\int_{B_{3}^{2}} r_{3}(\xi, x) d x\right]+\ldots \\
& =r_{0} P_{r}\left(S_{0}\right)+\int_{B_{1}} r_{1}(\xi, x) d x+\int_{B_{2}} r_{2}(\xi, x) d x+\int_{B_{3}} r_{3}(\xi, x) d x+\ldots \\
& =r\left(\xi ; T, D^{\circ}\right) .
\end{aligned}
$$

That is

$$
r\left(\xi ; S_{\xi}, D^{\sim}\right)>r\left(\xi ; T, D^{\circ}\right) \text {. }
$$

This contradicts with the fact that the sequdntial decision function $D_{\xi}=\left(S_{\xi}, D^{\circ}\right)$ is a Bayes solution relative to the a priori distribution $\xi$. Therefore the condition (iii) is necessary.

On the otherhand if the conditions (i), (ii) and (iii) are satisfied for the sequential decision function $D=(T, D)$, then we known from the above proof that $r(\xi, D)=r\left(\xi, D_{\xi}\right)$. Therefore in this case the sequential decision function $\underline{D}=(T, D)$ is a Bayes solution relative to $\xi$.

Consequently, the conditions (i), (ii) and (iii) are the necessary and sufficient condition, so that a sequential decision function may be a Bayes solution relative to the a pripri distribution $\xi$.

\section{References}

1) A. J. Arrow, D. Bhackwhlt, M. A. Grrshick; Bayes and Minimax solutions on Sequential decision problems, Eccnometrica, 17(1949), 213-244.

2) A. WAId and J. Wolfowitz; Bayes solutions of sequential decision problems. Ann, Math, Stat., 21(1950), 82-99; 